\title{
Perspectivas sobre la enseñanza de posgrado en Ciencias de la Documentación en España
}

Perspectives on the posgraduate and doctoral education in Library and Information Science in Spain

\author{
Francisco Javier García Marco \\ Universidad de Zaragoza, Facultad de Filosofía y Letras, \\ C/ Pedro Cerbuna 12, 50009 Zaragoza, jgarcia@unizar.es
}

\begin{abstract}
Resumen
Se analizan las implicaciones para la enseñanza superior en Ciencias de la Documentación del proceso de reforma de los ciclos formativos de la educación superior que está aconteciendo en España. Se compara la situación anterior y la actual, y se plantean tendencias, sugerencias y recomendaciones para el futuro. Se describe y explica la opción tomada por la Universidad de Zaragoza, enfocada a la enseñanza e investigación sobre gestión de unidades y servicios, y se contextualiza en un panorama dominado por los estudios orientados a la información digital - sin duda, el tema crítico en este momentoy por un sólido grupo de estudios dedicados a la Archivística, más minoritario.
\end{abstract}

Palabras clave: Ciencias de la Documentación. Enseñanza de posgrado. Enseñanza de doctorado. Tendencias. España.

\section{Introducción}

En los últimos diez años se ha acelerado el proceso de reforma de la educación superior española, que ha estado organizada bajo tres códigos legislativos distintos (España, Jefatura del Estado, 1983, 2001, 2007).

En el aspecto que ahora nos interesa -el diseño de las enseñanzas-, la compleja y debatida trayectoria discurre, sin embargo, por ciertas líneas comunes: búsqueda de la integración en el espacio europeo de educación superior necesario para promover la movilidad en el mercado europeo de trabajo (España, Ministerio de Educación, Cultura y Deporte, 2003abc)-, asunción de competencias por los poderes autonómicos en la educación superior, aumento de la autonomía de las universidades, expansión en el número de titulaciones oficiales —hasta su actual liberalización-.

\begin{abstract}
The implications of the new higher education cycle structure for the Library and Information Science LIS) instruction in Spain is analysed. The new situation is compared with the old one, and some trends, suggestions and guidelines are proposed for the future. The option chosen in the University of Zaragoza-oriented toward research and instruction on information and documentation units and services management-is described, explained and contextualized in a landscape mastered by posgraduate and doctoral studies oriented toward digital information-doubtless, the hottest topic today-and a solid but not so numerous group of studies on Archival Science.
\end{abstract}

Keywords: Library and Information Science. Posgraduate education. Doctoral education. Trends. Spain.

Los aspectos más notables del proceso de convergencia europea han sido la adopción de una unidad común de cuenta de la carga formativa -el crédito europeo del European Credit Transfer and Accumulation System (ECTS), desarrollado en el marco del programa de movilidad Sócrates-Erasmus-y de un marco común de organización de la educación superior, que adopta el modelo anglosajón de grado, máster y doctorado frente al anterior de licenciatura con diplomaturas-y doctorado. EI ECTS fue transpuesto al sistema nacional en 2003 (España, Ministerio de Educación, Cultura y Deporte, 2003). Los grados y posgrados están pendientes de normalización en un decreto ley del cual existe un borrador muy avanzado que cuenta con el consenso de la conferencia de rectores (España, Ministerio de Educación y Ciencia, 2007), después de unos decretos de transición entre el modelo anterior de titulaciones y el actual, promulgados durante el gobierno anterior 
(España, Ministerio de Educación y Ciencia, 2005ab).

Todo ello acontece en el marco de una importante ampliación de la oferta educativa para adultos - grados formativos superiores, nuevas carreras de primer y segundo ciclo, etc.-, de una notable mejora en la integración laboral de los jóvenes que los aleja de las aulas, del impacto de la bajada de la natalidad -y pronto de la emigración - en la matrícula universitaria y de la creciente especialización en el mercado de trabajo. Aspectos que han producido una gran reducción de los alumnos matriculados en carreras de biblioteconomía y documentación después de años de imparable expansión de estas titulaciones en las universidades españolas.

En este trabajo se van a revisar los cambios producidos y su implicaciones para la enseñanza de posgrado en documentación, y se presenta el trabajo realizado en la Universidad de Zaragoza.

\section{La enseñanza de posgrado en España y la introducción del nivel de máster}

La historia contemporánea de la educación superior en España arranca con la Ley de instrucción pública de 1857, conocida como Ley Moyano por haber sido promulgada en tiempos de Isabel II a instancias del Ministro de Fomento Claudio Moyano Samaniego. Esa ley es interesante para las ciencias de la documentación porque en ella se establece que academias, bibliotecas, archivos y museos quedan ligadas al sistema educativo (Título IV). Se trata de una ley que sitúa la cabecera del sistema universitario en Madrid, y que regula fuertemente todos los aspectos de la educación, incluyendo los grados -bachiller, licenciado y doctor-, las universidades, los títulos oficiales, los planes de estudio, etc. En los aspectos relacionados con la educación superior el objetivo que se lee entre líneas es, claramente, establecer las condiciones de acceso a las profesiones reguladas.

Esta ley estuvo vigente durante más de un siglo hasta 1970, año en el que se promulga la ley de reforma educativa de finales del franquismo (España, Jefatura del Estado, 1970). En esa época España era ya un estado industrial y el marco profesional del siglo había quedado desbordado. La enseñanza universitaria se organiza en tres ciclos: el primer ciclo dedicado a las disciplinas básicas, de tres años; el segundo ciclo, de especialización, con una duración de dos años; y un tercer ciclo de especialización concreta y preparación para la investigación y la docencia universitaria. El artículo 39, párrafo 4, abre la puerta a estudios de especialización no regulados por el Estado que cada universidad podría ofertar li- bremente: "Los estudios de especialización abiertos a los graduados universitarios de los distintos ciclos darán derecho a un certificado acreditativo de los mismos con los efectos profesionales que en cada caso se determinen."

En 1978 se promulga la constitución democráti$\mathrm{ca}$, que consagra las libertades de cátedra, estudio e investigación; reconoce a las universidades la autonomía en la gestión de sus recursos; y reparte las competencias en educación superior entre el Estado y las autonomías.

La ley socialista de 1983 (España, Jefatura del Estado, 1983) recoge las nuevas libertades $y$, entre otras cuestiones, reforma los gobiernos de las universidades y los cuerpos de profesores. En el aspecto que nos interesa, mantiene la estructura de ciclos de la ley de 1970 y una fuerte intervención en las titulaciones - a través del ya existente catálogo de títulos-, y de los currículos - a través de las directrices, que, sin embargo, se minimizan considerablemente-. Para su implantación, se exige el informe positivo en ambos niveles: el autonómico y el estatal.

La ley de reforma universitaria de 1983 daba también un paso más en la desregulación del sistema universitario español: El artículo 28 facultaba a las universidades para que "en uso de su autonomía" pudieran "impartir enseñanzas conducentes a la obtención de otros diplomas y títulos". Además, en el artículo 34, párrafo 4, la Ley de Reforma Universitaria concreta que las Universidades podrán establecer enseñanzas conducentes a la obtención de diplomas y títulos propios, así como enseñanzas de formación a lo largo de toda la vida, que, eso sí, carecerán de los efectos que las disposiciones legales otorgan a los títulos regulados. No se establecen programas económicos de apoyo.

Ante este nuevo panorama que se les abre, las universidades responden también dejando hacer: los profesores y grupos de profesores a través de diferentes órganos previstos en los estatutos proponen estudios de diversa duración que, por lo general, se consienten siempre que, como mínimo, se autofinancien. Se abre así, con todos sus defectos, así un interesantísimo experimento de libertad y responsabilidad dentro del encorsetado panorama universitario español, puesto que nacía un equilibrio natural entre oferta y demanda: los títulos que no se financiaban se cerraban. Lógicamente, el sistema quedaba abierto a la intervención a través de los mecanismos de subvención tanto para el sector privado como para la administración.

Ante tanta desregulación -inusitada en el panorama español del último siglo y medio-, un 
decreto aparecido el 1985 (España, Ministerio de Educación y Ciencia, 1985) intenta intervenir en ese amplio espacio creado por la ley. A pesar de todo, se limita a revindicar el derecho del Estado a intervenir en el ámbito de los títulos oficiales de posgrado -amparándose en la Ley de 1970-, a precisar que las universidades ofrecerán sus estudios propios a través de sus centros, a determinar que dichos estudios tendrán que ser "sobre campos del saber propios de la carrera de procedencia o de caracter intercurricular y especialmente orientadas a la aplicación profesional de dichos saberes", y a recordar que los diplomas no tendrán valor oficial "en tanto el gobierno no establezca lo contrario" (Artículo 17). Este último punto del texto demuestra la confusión del Estado y las Administraciones ante esta realidad surgida al margen de la tradición intervencionista del Estado en las Universidades y que estaba experimentando un notable éxito (1).

En 1987 aparece finalmente el real decreto que regulaba la homologación de títulos (Ministerio de Educación y Ciencia, 1987). Este documento vuelve a prestar especial atención a los títulos propios insistiendo en que no podrán inducir a confusión con los títulos oficiales y que no tendrán validez legal; estableciendo que las universidades deberán tener su registro de títulos propios expedidos; y regulando el camino para el reconocimiento de los títulos como oficiales a través de su aprobación en el Consejo de Universidades motu proprio o a propuesta de cualquier universidad.

Por otra parte, los estudios de doctorado se encontraban plenamente desregulados en lo concerniente a título y contenidos específicos, que ha sido la tendencia que se está imponiendo en el resto de los niveles, y especialmente el de máster.

En 1998 el nuevo gobierno liberal-conservador publica un decreto reformando el tercer ciclo, por el que, entre otras cosas, se establece un título intermedio en el doctorado -el diploma de estudios avanzados-con la esperanza de atender la demanda de formación en la investigación especializada y de captar estudiantes para los programas de doctorado establecidos en el 1983, ofreciendo a los estudiantes un reconocimiento a mitad del proceso. Este movimiento debe entenderse también con el transfondo de la competencia de los títulos propios en la captación de alumnos de tercer ciclo, que el Estado y las universidades contemplan con ambivalencia independientemente de la tendencia política. También refleja la profunda insatisfacción con el doctorado, al que se está dedi- cando mucho profesorado para relativamente pocos alumnos y muchas menos tesis.

En 2001, los liberal-conservadores promulgan su propia ley de educación superior (España, Jefatura del Estado, 2001). Esta ley recoge la reflexión del proceso de Bologna y el énfasis en la necesidad de formación avanzada y permanente en el marco de los "vertiginosos cambios" que está conllevando la sociedad del conocimiento. En el aspecto que nos interesa -el del marco curricular-, el Estado sigue reservándose, como en la ley socialista de 1983, la aprobación de títulos oficiales recogidos en el mencionado catálogo y de las directrices curriculares básicas (Art. 33). Se mantiene la autonomía de las universidades para crear "diplomas y títulos propios, así como enseñanzas de formación a lo largo de toda la vida" sin los efectos de los títulos homologados (párrafo 3). Respecto a las titulaciones oficiales, el modelo es aproximadamente el mismo: titulaciones en tres ciclos (diplomado-técnico superior, licenciado-arquitectoingeniero, doctor). En cuanto al proceso, las universidades proponen, las autonomías aprueban la memoria económica de los estudios, el Estado comprueba la conformidad con las directrices, y una agencia de evaluación y acreditación comprueba a posteriori el desempeño. En el año 2004, se publica el real decreto que regula la homologación de títulos oficiales.

En el 2005 se publican sendos reales decretos (España, Ministerio de Educación y Ciencia, 2005abc) que establecen la estructura de las enseñanzas universitarias y se regulan los estudios de grado y posgrado. El objetivo es implantar el modelo establecido en la conferencia de ministros responsables de la educación superior en la Comunidad Europea celebrada Berlin en 2003. Dicho modelo establece dos niveles grado y posgrado- , que se dividen en conjunto en tres ciclos (España, Ministerio de Educación y Ciencia, 2005a):

El primer nivel, o de Grado, comprende las enseñanzas universitarias de primer ciclo y tiene como objetivo lograr la capacitación de los estudiantes para integrarse directamente en el ámbito laboral europeo con una cualificación profesional apropiada. El segundo nivel, comprensivo de las enseñanzas de Posgrado, integra el segundo ciclo de estudios, dedicado a la formación avanzada y conducente a la obtención del título de Máster, y el tercer ciclo, conducente a la obtención del título de Doctor, que representa el nivel más elevado en la educación superior.

El decreto de posgrado (España, Ministerio de Educación y Ciencia, 2005b) especifica que "El segundo ciclo de los estudios universitarios estará dedicado a la formación avanzada, de 
carácter especializada o multidisciplinar, dirigida a una especialización académica o profesional o bien a promover la iniciación en tareas investigadoras. La superación del ciclo dará derecho a la obtención del título de Máster." Los estudios tendrán una duración entre 60 y 120 créditos, las universidad podrán crear especialidades dentro de ellos, y en el caso de las profesiones reguladas, el Estado podrá proponer requisitos mínimos.

Ese mismo año el Partido Socialista vuelve al poder y una de sus primeras acciones en materia educativa es corregir los decretos anteriores de 2005, para acomodar los recursos presentados por la Generalitat catalana (España, Ministerio de Educación y Ciencia, 2005c). Como resultado, la implantación de los estudios oficiales de posgrado será aprobada por la comunidad autónoma competente, que determinará también la agencia de evaluación competente, mientras que la impartición de los másteres oficiales requerirá homologación por el Estado.

En abril de 2007, el Ministerio de Educación y Ciencia (España, Ministerio de Educación y Ciencia, 2007) modifica la ley de 2001. En los aspectos relacionados con la estructura de las titulaciones, los ciclos pasan a ser grado, máster y doctorado (Artículo 37). Se mantiene la distinción entre títulos oficiales con validez en todo el territorio nacional —que deberán inscribirse en el Registro de universidades, centros y títulos, y con directrices establecidas por el Gobierno - y enseñanzas conducentes a la obtención de otros títulos, que podrán inscribirse a efectos informativos. Los títulos serán aprobados por las comunidades autónomas, respetando la autonomía de las universidades, y verificados a priori por el Consejo de Universidades y a posteriori por las agencias de calidad universitaria.

El decreto que regula la estructura de las enseñanzas está en fase de consultas finales y ha recibido el visto bueno de la conferencia de rectores y los agentes sociales. Desaparecen las denominaciones establecidas para los títulos: en el grado se establecen ramas dentro de las cuales cada universidad podrá proponer su título; en el máster tan solo el título del estudio y la universidad; y en el doctorado la universidad con información del campo en el que se ha realizado la tesis. La finalidad de las enseñanzas de máster queda establecidad en "la adquisición por el estudiante de una formación avanzada, de carácter especializado o multidisciplinar, orientada a la especialización académica o profesional, o bien a promover la iniciación en tareas investigadoras". Tras un grado de 240 créditos y un tronco común de 60 créditos por macroárea científica —que alarga la formación superior respecto a la media europea-, el máster tendrá entre 60 y 120 créditos, con un mínimo de 6 y un máximo de 30 para un trabajo de fin de máster. El plan de estudios es por lo demás libre, salvo en lo que respecta a la consecución de las competencias establecidas para las profesiones reguladas, que no es el caso de la Biblioteconomía y Documentación. Se accederá con al menos 240 créditos, por lo que los antiguos diplomados deberán cursar los créditos adicionales. Los estudios de máster dan acceso al doctorado con sesenta créditos en uno o varios másteres, y el doctorado se limita básicamente a la realización de la tesis doctoral. Los títulos serán re-acreditados cada seis años.

Así pues, la historia de la educación superior desde la implantación de la democracia es, fundamentalmente, la historia de la convergencia europea en esta materia. Como resultado, se ha impuesto el modelo grado (bachillerato o licenciatura), máster y doctorado, propio del ámbito anglosajón, y adoptado en bastantes países europeos en los últimos años (Dinamarca en 1993, Italia y Suiza en 1999, Alemania, Austria, Benelux y Noruega en 2002, Francia en 2003). El modelo anglosajón de autonomía universitaria en lo que se refiere a los títulos se ha ido concretando a lo largo de la democracia en uno que funciona en tres fases: en la primera, cada universidad propone a partir de sus mecanismos internos; en la segunda, se realiza una supervisión previa en los tres niveles -universidad, autonomía y estado-; y, en la tercera, se asegura una supervisión a posteriori a través de las agencias de calidad y acreditación, que se supone que retroalimenta el proceso a nivel de la universidad. El estado central y autonómico se reservan una importante vía de intervención a través de los contratos ligados a resultados y los planes de incentivación.

Se conforma, por tanto, un sistema mixto en el que, por una parte, se individualizan los actores (esto es, las universidades y sus unidades) que prestan los servicios educativos superiores para facilitar el ajuste a la demanda educativa y la asunción de responsabilidades; y, por la otra, los poderes públicos -fundamentalmente los autonómicos - pueden intervenir si lo desean a través de la financiación.

Sin descartar la formación específica, el doctorado vuelve a ser básicamente la realización de la tesis. Se abre el camino para que los títulos propios y periodos formativos oficiales existosos puedan ser reconvertidos en másteres oficiales, simplificando el panorama considerablemente. La formación en la investigación y la especialización se devuelve al segundo ciclo, en la línea de lo pretendido ya en la ley de 1970. 


\section{El momento actual}

El momento actual se puede caracterizar como un periodo de transición entre el modelo anterior de posgrado "estudio propio" y el modelo futuro de máster de libre configuración, con objetivos de especialización profesional o de iniciación a la investigación y puerta del doctorado.

En España se ha dado el caso de que existían estudios de Diplomatura en Biblioteconomía y Documentación, un estudio de segundo ciclo en Documentación, y estudios propios con nivel de máster. Estos últimos han tenido fundamentalmente carácter de especialización, como el Máster en Información y Documentación que, desde 1992, se impartía en la Universidad Carlos III de Madrid con la colaboración del Consejo Superior de Investigaciones Científicas.

Los centros y departamentos universitarios españoles han recibido bien el proceso de Bolonia y publicaron conjuntamente un proyecto de reforma de los estudios de información y documentación conocido como libro blanco (Agencia Nacional de Evaluación de la Calidad y Acreditación, 2004). En él recogen su acuerdo básico sobre el segundo ciclo (ibidem, p. 31):

Se propone que los estudios de máster tengan una duración de entre 60 y 120 créditos en función de la procedencia de los alumnos y los requerimientos que ponga cada universidad. Se recomienda que los programas de máster que se organicen puedan tener una doble orientación, profesional e investigadora. Esta segunda línea facilitará que aquellos alumnos que lo deseen puedan iniciar un programa de doctorado al finalizar 300 créditos.

En ese mismo documento se recoge el interés de las asociaciones de archiveros por un máster específico.

\section{Un análisis de los posgrados existentes}

En la tabla I, en la página siguiente, se presenta el resultado de una investigación realizada en otoño de 2006 como parte de la preparación del estudio de viabilidad del programa oficial de posgrado de la universidad de Zaragoza. Se han añadido posteriormente los másteres aprobados en la Universidad de Alcalá, Universidad de Salamanca y en la de Zaragoza.

La distribución geográfica se presenta en la figura 1 . Aunque se trata de estudios muy heterogéneos, es posible hacerse una idea de los centros geográficos más activos y con más demanda (Madrid, Valencia, Andalucía y Cataluña).

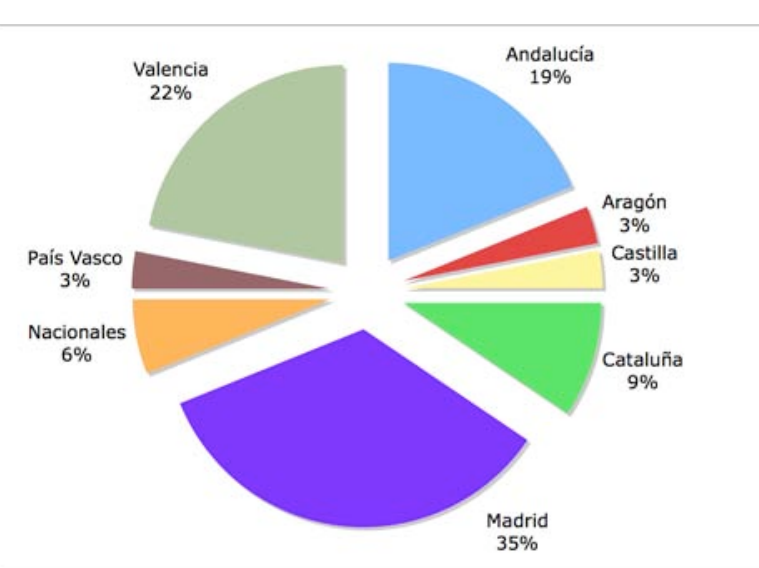

Figura 1. Distribución por comunidades autónomas

En cuanto a las universidades, la más activa es la Complutense con cinco estudios, la Internacional de Andalucía con 3, y las Carlos III de Madrid, Sevilla, Valencia y UNED con dos estudios cada una.

Si atendemos al tipo de estudios, la mayoría son de breve duración - del tipo diplomas de especialización-, especialmente en las universidades privadas, que encuentran así un nicho de mercado diferenciado. Los másteres LRU son muy pocos frente a los posgrados -normalmente las universidades adoptan esta terminología para distinguir los estudios de 90 o 120 créditos frente a los que rondan los 60 - y los másteres oficiales están sido rápidamente adoptados, aunque son muy variados en cuanto a su duración en créditos, dentro de la horquilla de 60 a 120 créditos.

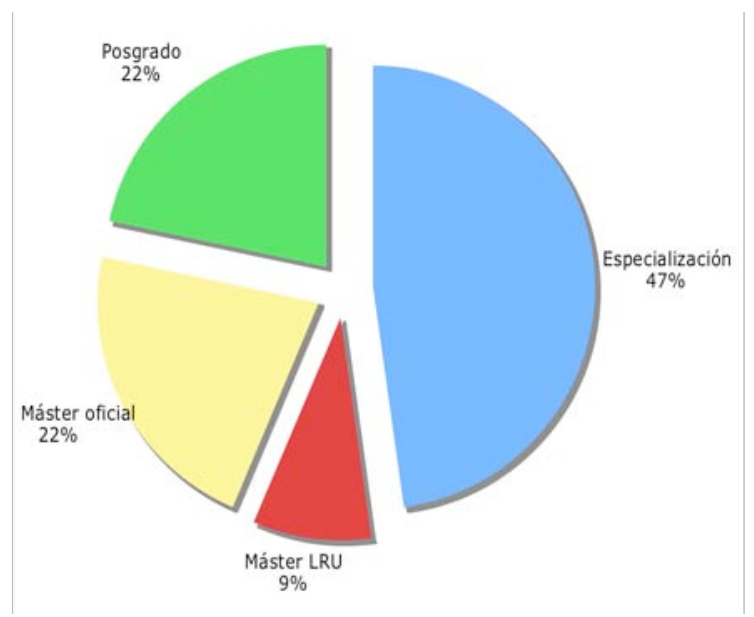

Figura 2. Tipos de estudios 


\begin{tabular}{|c|c|}
\hline Título del estudio de posgrado & Universidad \\
\hline Certificado de especialización en bibliotecas escolares e infantiles & U. de Vic \\
\hline Curso de postgrado de especialista universitario en archivísitica & Fundación Carlos de Amberes \\
\hline $\begin{array}{l}\text { Diploma de especialización en documentación y gestión del patrimonio } \\
\text { histórico. Archivos, bibliotecas, museos }\end{array}$ & U. de Deusto \\
\hline Diploma de posgrado en documentación digital on line & $\begin{array}{l}\text { Universidad Pompeu Fabra de } \\
\text { Barcelona }\end{array}$ \\
\hline Diploma de postgrado en documentación y sistemas de información sanitaria & Fundació Doctor Robert \\
\hline Diploma universitario en recuperación y documentación de la música sacra & U. San Vicente Mártir \\
\hline Especialista en historia de la bibliografía y documentación jurídicas & U. Complutense de Madrid \\
\hline $\begin{array}{l}\text { Especialista en información digital en medios de comunicación social (on- } \\
\text { line) }\end{array}$ & U. Complutense \\
\hline Especialista universitario en Archivística & Fundación Carlos de Amberes \\
\hline Especialista universitario en Archivística & UNED \\
\hline Experto en gestión documental & U. Internacional de Andalucía \\
\hline $\begin{array}{l}\text { Experto en gestión y tratamiento de la información en instituciones públicas y } \\
\text { privadas }\end{array}$ & U. Complutense \\
\hline Experto en información y documentación ambiental & U. Complutense \\
\hline Experto en información, documentación y bibliotecas de Ciencias de la Salud & U. Internacional de Andalucía \\
\hline Experto universitario en Biblioteconomía y Documentación & UNED \\
\hline Máster de Biblioteconomía (a distancia) & $\begin{array}{l}\text { ETD. Estudio de Técnicas } \\
\text { Documentales }\end{array}$ \\
\hline Máster en Archivística & U. Carlos III de Madrid \\
\hline Máster en Archivística & U. de Sevilla \\
\hline Máster en archivos y gestión documental & U. de Cádiz \\
\hline Máster en bibliotecas & U. de Sevilla \\
\hline Máster en documentación médica & U. de Valencia \\
\hline Máster en documentación y sistemas de información sanitaria & U. de Valencia \\
\hline Máster en gestión de bibliotecas, archivos y centros de documentación & $\begin{array}{l}\text { Escuela de Negocios San Pablo CEU } \\
\text { de Valencia }\end{array}$ \\
\hline Máster en gestión de contenidos digitales & U. de Barcelona \\
\hline Máster en gestión documental y administración de archivos & $\begin{array}{l}\text { Universidad Internacional } \\
\text { de Andalucía }\end{array}$ \\
\hline Máster en promoción de la lectura & U. de Alcalá \\
\hline Máster oficial en bibliotecas & U. Complutense \\
\hline Máster oficial en información científica: tratamiento, acceso y evaluación & U. de Granada \\
\hline $\begin{array}{l}\text { Máster oficial en investigación en documentación: documentación } \\
\text { audiovisual: gestión del conocimiento en el entorno digital }\end{array}$ & U. Carlos III de Madrid \\
\hline $\begin{array}{l}\text { Máster oficial en planificación y gestión de unidades y servicios de } \\
\text { información y documentación }\end{array}$ & U. de Zaragoza \\
\hline Máster oficial en sistemas de información digital & U. de Salamanca \\
\hline Máster universitario en bibliotecas digitales & U. de Valladolid \\
\hline Postgrado en gestión de los recursos digitales en la empresa & U. Jaume I \\
\hline
\end{tabular}

Tabla I. Estudios de posgrado en España.

En cuanto a las especialidades (Tablas II y III, figuras 3 y 4 ) el grupo más compacto son los estudios de archivística. La información digital también es muy popular, aunque no es tan compacto temáticamente. Algunos estudios de este grupo son eminentemente informáticos, como el posgrado en bibliotecas digitales impartido en la Universidad de Valladolid. El grupo dedicado a las bibliotecas es menos consistente y sorprendentemente reducido, con dos estu- dios dedicados a la animación de la lectura y las bibliotecas escolares, infantiles y juveniles. Por fin, el dedicado a las documentaciones especializadas es también numeroso, con un subgrupo muy importante dedicado a las documentaciones sanitarias, en las que las universidades valencianas destacan fuertemente. Solo dos universidades abordan los aspectos relacionados con la gestión. Queda, por fin, algún estudio aislado de tipo generalista. 


\begin{tabular}{|l|l|}
\hline Especialidad & $N^{\circ}$ \\
\hline Archivos & 7 \\
\hline Bibliotecas & 3 \\
\hline Bibliotecas digitales & 1 \\
\hline Bibliotecas juveniles & 2 \\
\hline Documentación ambiental & 1 \\
\hline Documentación audiovisual & 1 \\
\hline Documentación digital & 3 \\
\hline Documentación jurídica & 1 \\
\hline Documentación médica & 4 \\
\hline Documentación musical & 1 \\
\hline Generalista & 1 \\
\hline Gestión de contenidos & 3 \\
\hline Gestión de la información & 1 \\
\hline Interdisciplinar & 1 \\
\hline Información científica & 1 \\
\hline Gestión & 2 \\
\hline
\end{tabular}

Tabla II. Clasificación temática analítica.

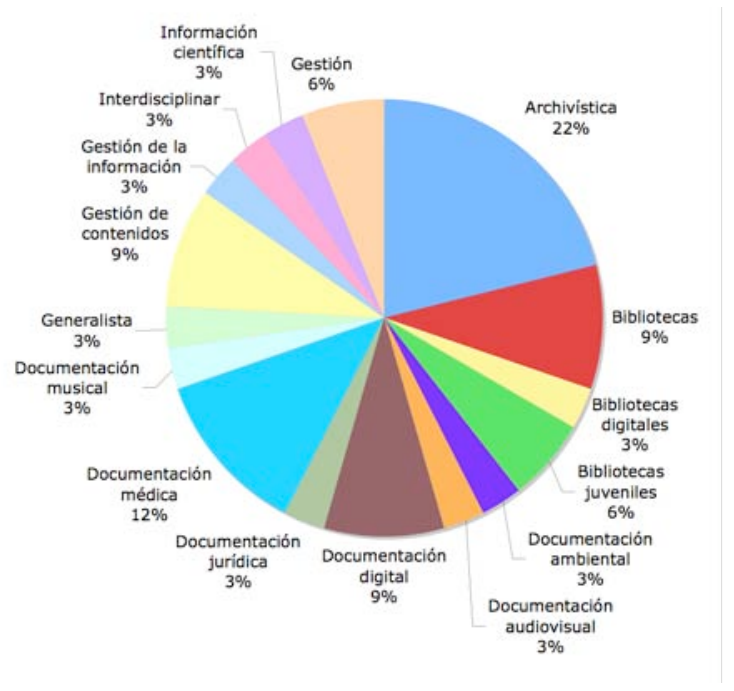

Figura 3. Clasificación temática analítica

\begin{tabular}{|l|l|}
\hline Especialidad & $N^{\circ}$ \\
\hline Archivos & 8 \\
\hline Bibliotecas & 5 \\
\hline Información digital & 8 \\
\hline Documentaciones especializadas & 8 \\
\hline Gestión & 2 \\
\hline Generalista & 1 \\
\hline Interdisciplinar & 1 \\
\hline
\end{tabular}

Tabla I.II. Clasificación temática sintética.

La diversidad, pues es importante, pero archivística - un nicho profesional importante que requiere formación específica- e información digital —el signo de los tiempos- ocupan prác- ticamente la mitad de la oferta. Hay pocos estudios que aborden el tema de la preparación de cuadros superiores, con formación en técnicas de gestión modernas. Las documentaciones especializadas están, sobre todo, sesgadas al área biomédica. La diversidad en la formación de posgrado en el campo de las bibliotecas es reducida. No hay másteres especializados en bibliotecas públicas ni universitarias, aunque sí que se atiende a la animación a la lectura. En la misma dirección, habiendo tantos másteres en archivos, sería interesante una diversificación en los diferentes tipos de archivos, pero eso no ha ocurrido.

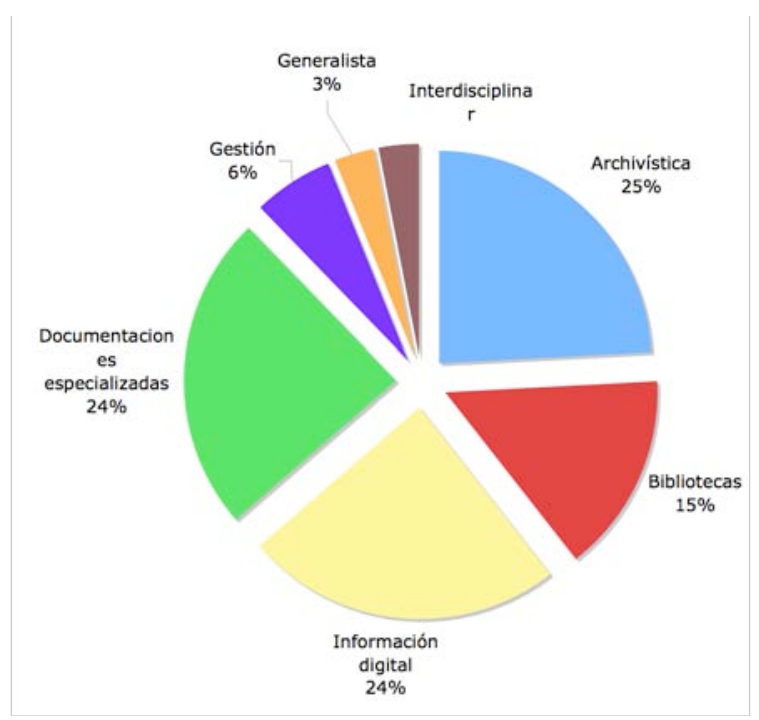

Figura 4. Clasificación temática analítica.

Así pues, el panorma de los másteres en nuestro país no está suficientemente artículado para dar cuenta de la demanda. Una intervención adecuada requeriría un cuidadoso estudio de otros parámetros fundamentales como el número de créditos, estudiantes, profesores, etc.

\section{El máster de la Universidad de Zaragoza}

EL Departamento de Ciencias de la Documentación e Historia de la Ciencia de la Universidad de Zaragoza - dirigido entonces por el Prof. Genaro Lamarca- respondió rápido a la posibilidad de ofrecer un programa oficial de posgrado, y presentó un proyecto al Consejo de Gobierno a finales de 2006. El proceso fue coordinado por el autor en un proceso participativo organizado en forma de dos círculos concéntricos: el interno, de coordinación, constituido por los profesores Carmen Agustín, Genaro Lamarca, José Antonio Salvador y Esperanza Velasco; 
y el amplio, formado por todo el Departamento. Fue aprobado en abril de 2007 y comenzará a impartirse en octubre de este año.

\subsection{Planificación estratégica}

Tras un análisis tipo DAFO, se establecieron los objetivos del programa: distinguirse adecuadamente del grado en objetivos, nivel y contenidos; diferenciarse de la oferta de las demás universidades - centradas en la archivística, la información digital y cubriendo las bastantes especialidades de la documentación-; ofrecer formación diversificada a una base local reducida -Aragón es una región poco poblada y envejecida-; resultar atractivo a estudiantes procedentes de un amplio marco geográfico; aprovechar un cuerpo de profesores de diversas especialidades; y favorecer la cohesión intradepartamental ante los nuevos retos.

A partir de estos requisitos, se optó por centrar los estudios en el campo de la planificación y gestión de unidades y servicios de información y documentación, con tres especialidades: archivos, bibliotecas y documentación especializada.

El reto se definió como formar especialistas en el nivel de técnico superior en bibliotecas, archivos, centros de información y documentación, y gestión de contenidos que demandan las administraciones y las empresas, así como de investigadores capaces de animar el tejido de transferencia del conocimiento socialmente acumulado a través de una investigación de calidad y el desarrollo de nuevos productos y servicios innovadores en su entorno, y, por ende, de integrarse en el programa de doctorado.

En el nivel de máster, el programa se orienta específicamente a la formación de tres tipos de perfiles:

- personal técnico superior especializado en la gestión y dirección de archivos, bibliotecas, centros de documentación y repositorios de contenidos,

- investigadores capaces de integrarse en el programa de doctorado, y...

- responsables del desarrollo de productos y servicios de información y documentación innovadores en su entorno.

Se previó una doble demanda: graduados en Biblioteconomía y Documentación, y titulados procedentes de otras especialidades que desearan reorientar su currículos. A los graduados en Biblioteconomía y Documentación, se les pretende aportar fundamentalmente la formación en gestión y la perspectiva gerencial necesaria para promocionar su carrera profesional, así como una solida introducción a la investigación, necesaria para la resolución de problemas nuevos y para progresar, si lo desean, hacia una carrera propiamente investigadora. Por otra parte, a los titulados de otras especialidades, se busca proporcionales una excelente salida profesional muy demanda ajustando su formación a campos profesionales interesantes -archivero, bibliotecario, documentalista- que se benefician extraordinariamente de una formación especializada complementaria - Historia, Humanidades, Medicina, Química, etc.- , tanto en lo que se refiere al ejercicio profesional como al propiamente académico e investigador.

En el nivel de doctorado, el programa se orienta a la formación de investigadores científicos autónomos, responsables, innovadores, críticos y capaces de integrarse en equipos de investigación disciplinares e interdisciplinares en el campo de la información y documentación.

El programa de estudios (Apéndice) combina los contenidos avanzados en gestión de la información documental, contenidos en administración moderna y contenidos especializados, con un fuerte énfasis en la realización de prácticas, trabajos de investigación y memorias profesionales.

\subsection{Perfil formativo: competencias}

Se persigue el desarrollo de las siguientes competencias transversales:

- Planificar, diseñar, desarrollar y evaluar productos, unidades y servicios de información.

- Diseñar, gestionar y controlar proyectos en el campo de la información y la documentación.

- Conocer, interpretar y aplicar el marco jurídico y deontológico de la actividad documental.

- Analizar y comprender el contexto de las unidades y servicios de información documental.

- Comprender e intervenir en los procesos de evaluación, conservación, representación y organización de la información y el conocimiento.

- Conocer y utilizar las tecnologías de la información y la comunicación en la mejora y automatización de las unidades y servicios de información y documentación.

- Atender al factor humano en la gestión de unidades y servicios de información y documentación tanto en el lado de la demanda (usuarios) como de la oferta (profesionales).

- Diseñar y efectuar investigaciones científicas con metodologías cualitativas y cuantitativas 
en el campo de la información y la documentación.

Esas competencias se aplican a los principales campos profesionales de la información y la documentación: los archivos de la administración estatal, autonómica y local; los archivos de las organizaciones empresariales y entidades y personas privadas; las bibliotecas públicas, nacionales, autonómicas, universitarias y de investigación, escolares, infantiles y juveniles y a las bibliotecas y colecciones históricas; y los centros de documentación médica y científicotécnica, jurídica y económico-empresarial, administrativa, informativa, y en humanidades y patrimonio histórico.

\subsection{Plan de estudios}

Se ha optado por una modularización en cursos de tres créditos que se imparten de forma secuencial y monográfica en bloques de dos semanas. Se facilita así el proceso de transferencia del conocimiento entre estudiantes y profesores y el aprendizaje autónomo por parte de estudiantes adultos, a la vez que el nivel de detalle permite un control riguroso de las expectativas de los estudiantes y de la calidad de los cursos y procesos de aprendizaje. Las asignaturas en ningún caso tendrán nivel de introducción, sino que se ajustarán en general a los objetivos previstos en los descriptores de Dublín para el nivel de máster y específicamente en el título. Sin embargo, los profesores han adquirido la responsabilidad de prever un itinerario de formación previo para los estudiantes que procedan de grados diferentes del de Biblioteconomía y Documentación, de manera que puedan seguir los cursos de forma adecuada con un trabajo suplementario razonable.

Los estudiantes de maestría realizarán prácticas relacionadas con la planificación, desarrollo e implantación de un nuevo producto o servicio de información y documentación, o bien con la auditoría y evaluación de uno existente en el marco de la asignatura Prácticas especializadas, de las cuales presentarán una memoria. Dicho trabajo de campo se completará con un estado de la cuestión científico-técnico sobre los diferentes aspectos del problema elegido, que realizarán, fuera del centro de prácticas, en el marco de la asignatura Trabajos de investigación.

La secuencia del proceso de las Prácticas especializadas, diseñada por el autor, se compone de nueve fases: a) toma de contacto con la unidad estudiada; b) realización de un análisis DAFO de la unidad; c) propuesta de ámbitos de intervención; d) selección de un proyecto de intervención de mutuo acuerdo entre el estudiante, el responsable de la unidad (tutor profesional) y el profesor tutor de prácticas (tutor académico); e) realización del proyecto de intervención bajo la doble supervisión del tutor profesional y académico; g) elaboración del informe del proyecto de intervención; $h$ ) visto bueno de los responsables profesional y académico; y i) calificación por el tutor académico.

En la trayectoria final del máster, los estudiantes realizarán bajo la dirección de un director académico de su elección un Proyecto de investigación que deberá recoger de forma coherente, sistemática y justificada su proyecto de prácticas especializadas, sus trabajos de investigación monográficos y la tesis de maestría que dará culminación al máster y servirá para la presentación pública y la socialización de los resultados del aprendizaje. El proyecto de investigación deberá tomar la forma de un documento formal, ser conocido por el Consejo de Coordinación del Máster y ser archivado durante al menos cinco años en el Archivo del Departamento.

Los Trabajos de investigación consistirán en una o varias investigaciones científicas relacionadas con cinco asignaturas entre las cursadas durante los dos primeros semestres que sean del especial interés del maestrando bajo la dirección de los profesores responsables de las mismas y dentro del citado proyecto de investigación acordado con su director académico.

La Tesis de maestría será defendida públicamente ante un tribunal de tres doctores (como mínimo dos) o profesionales de reconocido prestigio con nivel de Licenciatura o Máster que tengan cargos directivos en centros o servicios con un equipo superior a cinco personas de plantilla, seleccionados a propuesta del Consejo de Coordinación, oído el Director de la tesis de maestría. Dicho Director no podrá formar parte de dicho tribunal.

\section{Conclusiones}

Es obvio que en el contexto actual de globalización, tecnologías de formación a distancia y de abundantes recursos para movilidad, las diferentes universidades deberían especializarse en diferentes tipos de estudios utilizando los mejores recursos humanos de las diferentes universidades españolas y extranjeras. Sin embargo, a la vista de los resultados preliminaries, no está claro que se estén consiguiendo estos objetivos, formulados inicialmente ya a comienzos de los años sententa. A pesar de todo, el nuevo marco legal debe valorarse de forma muy positiva, pues ofrece amplio campo a la innovación e 
incrementa las posibilidades de diversificación y reorganización del mapa universitario. Los agentes deben cooperar para mantener una comunicación fluida que preserve un marco común y favorezca la generalización de buenas prácticas, pero deberían evitar una concertación en cuanto a los títulos y su estructura, pues de lo contrario se perderá buena parte de las posibilidades ofrecidas. Antes bien, deberían centrarse en identificar su misión específica dentro de la ecología de la formación en ciencias de la documentación, y ajustarse a los mercados seleccionados de manera realista y científica.

\section{Notas}

(1) Es interesante señalar que el Instituto Nacional de Estadística no ofrece datos sobre la evolución de estos estudios; en parte, sin duda, por la dificultad que plantea la inexistencia de un registro único de títulos.

\section{Referencias}

Agencia Nacional de Evaluación de la Calidad y Acreditación (2004). Libro blanco. Título de grado en Información y Documentación. Madrid: ANECA, 2004. Disponible en http://www.aneca.es/modal_eval/docs/libroblanco_jun05 documentaci \%F3n.pdf. (Consulta 25/06/2006).

España, Jefatura del Estado (1970). Ley 14/1970, de 4 de agosto (Jefatura), General de Educación y Financiamiento de la Reforma Educativa. // Boletín Oficial del Estado. 187 (6/8/1970). 12525-12546.

España, Jefatura del Estado (1983). Ley Orgánica 11/1983, de 25 de agosto, de Reforma Universitaria. // Boletín Oficial del Estado. 209 (1/9/1983) 24034-24042.

España, Jefatura del Estado (2001). Ley Orgánica 6/2001, de 21 de diciembre, de Universidades. / /Boletín Oficial del Estado. 307 (24/12/2001) 49400-49425.

España, Jefatura del Estado (2007). Ley Orgánica 4/2007, de 12 de abril, por la que se modifica la Ley Orgánica $6 / 2001$, de 21 de diciembre, de Universidades. 89 (13/4/2007) 16241-16260

España, Ministerio de Educación y Ciencia (1985). Real Decreto 185/1985, de 23 de enero, por el que se regula el tercer ciclo de estudios universitarios, la obtención y expedición del titulo de doctor y otros estudios postgraduados. // Boletín Oficial del Estado. 41 (16/2/1985). 3947-3953.

España, Ministerio de Educación y Ciencia (1987). Real Decreto 1496/1987, de 6 de noviembre, sobre obtención, expedición y homologación de títulos universitarios. I/ Boletín Oficial del Estado. 298 (14/12/1987). 36637-36639

España, Ministerio de Educación y Ciencia (2005c). Real Decreto $1509 / 2005$, de 16 de diciembre, por el que se modifican el Real Decreto 55/2005, de 21 de enero, por el que se establece la estructura de las enseñanzas universitarias y se regulan los estudios universitarios oficiales de grado y el Real Decreto 56/2005, de 21 de enero, por el que se regulan los estudios universitarios oficiales de posgrado. // Boletín Oficial del Estado. 303 (20/12/2005) 41455-41457.
España, Ministerio de Educación y Ciencia (2005a). Real Decreto 55/2005, de 21 de enero, por el que se establece la estructura de las enseñanzas universitarias y se regulan los estudios universitarios oficiales de Grado. // Boletín Oficial del Estado. 21 (25/1/2005) 2842-2846.

España, Ministerio de Educación y Ciencia (2005b). Real Decreto 56/2005, de 21 de enero, por el que se regulan los estudios universitarios oficiales de posgrado. // Boletín Oficial del Estado. 21 (25/1/2005) 2846-2851.

España, Ministerio de Educación y Ciencia (2007). Real Decreto por el que se establece la ordenación de las enseñanzas universitarias oficiales: borrador. Madrid: Ministerio de Educación y Ciencia, 26-06-2007. http://www.mec.es/mecd/gabipren/documentos/files/200 7-rd-ensenanzas-borrador.pdf.

España, Ministerio de Educación y Cultura (1998). Real Decreto 778/1998, de 30 de abril, por el que se regula el tercer ciclo de estudios universitarios, la obtención y expedición del titulo de doctor y otros estudios de postgrado. // Boletín Oficial del Estado. 104 (1/5/1998). 1468814696.

España, Ministerio de Educación, Cultura y Deporte (2003a). Real Decreto 1044/2003, de 1 de agosto, por el que se establece el procedimiento para la expedición por las universidades del Suplemento Europeo al Título. // Boletín Oficial del Estado. 218 (11/9/2003) 33848-33853.

España, Ministerio de Educación, Cultura y Deporte (2003b). Real Decreto 1125/2003, de 5 de septiembre, por el que se establece el sistema europeo de créditos y el sistema de calificaciones en las titulaciones universitarias de carácter oficial y validez en todo el territorio nacional. // Boletín Oficial del Estado. 224 (18/9/2003) 34355-34356.

España, Ministerio de Educación, Cultura y Deporte (2004). Real Decreto 49/2004, de 19 de enero, sobre homologación de planes de estudios y títulos de carácter oficial y validez en todo el territorio nacional. // Boletín Oficial del Estado. 19 (22 enero 2004) 2667-2671

España, Ministerio de Fomento (1857). Ley de Instrucción pública autorizada por el Gobierno para que rija desde su publicación en la Península é Islas adyacentes, lo que se cita. // Gaceta de Madrid. 1710 (10/09/1957) 13.España, Jefatura del Estado (1983). Ley Orgánica 11/1983, de 25 de agosto, de Reforma Universitaria. /Boletín Oficial del Estado. 209 (1/9/1983) 24034-24042

España, Ministerio de la Presidencia (2003). Real Decreto $1171 / 2003$, de 12 de septiembre, por el que se incorpora al ordenamiento jurídico español la Directiva 2001/19/CE del Parlamento Europeo y del Consejo, de 14 de mayo de 2001, por la que se modifican directivas sobre reconocimiento profesional, y se modifican los correspondientes reales decretos de transposición. // Boletín Oficial del Estado. 225 (19/9/2003) 34457-34498. 


\section{Apéndice: plan de estudios}

\begin{tabular}{|c|c|c|c|c|c|}
\hline Materia & Objetivos de aprendizaje & 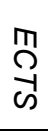 & 궁 & 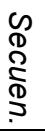 & Carácter \\
\hline $\begin{array}{l}\text { Metodología de la } \\
\text { investigación científica }\end{array}$ & $\begin{array}{l}\text { Comprender y aplicar el concepto de saber científico, las fases de una } \\
\text { investigación científica y las principales herramientas necesarias para } \\
\text { llevar a cabo una investigación científica. }\end{array}$ & 3 & OB & 1 & Metodológica \\
\hline $\begin{array}{l}\text { Técnicas cuantitativas } \\
\text { aplicadas a la } \\
\text { investigación en } \\
\text { Documentación }\end{array}$ & $\begin{array}{l}\text { Adquirir los conocimientos, actitudes y habilidades en el manejo del } \\
\text { SPSS como herramienta de trabajo en la investigación y la aplicación } \\
\text { de los tests estadísticos adecuados a los problemas planteados en la } \\
\text { gestión de unidades y servicios de información y documentación. }\end{array}$ & 3 & OB & 1 & Metodológica \\
\hline $\begin{array}{l}\text { Marco jurídico y } \\
\text { deontológico de la } \\
\text { actividad documental }\end{array}$ & $\begin{array}{l}\text { Conocer, interpretar y aplicar la legislación y los principios y códigos } \\
\text { deontológicos en el contexto de las unidades y servicios de información } \\
\text { y documentación }\end{array}$ & 3 & $\mathrm{OB}$ & 1 & $\begin{array}{l}\text { Teórica- } \\
\text { Aplicada }\end{array}$ \\
\hline $\begin{array}{l}\text { Planificación, diseño y } \\
\text { desarrollo de servicios de } \\
\text { información }\end{array}$ & $\begin{array}{l}\text { Conocer el ciclo de vida de un servicio de información documental y } \\
\text { saber } \\
\text { aplicar la metodología para su adecuada gestión, con especial } \\
\text { incidencia en } \\
\text { el entorno digital }\end{array}$ & 3 & OB & 1 & $\begin{array}{l}\text { Teórica- } \\
\text { Aplicada }\end{array}$ \\
\hline $\begin{array}{l}\text { Gestión de proyectos en } \\
\text { servicios de información }\end{array}$ & $\begin{array}{l}\text { Ser capaz de planificar y gestionar las distintas variables implicadas en } \\
\text { el desarrollo de un proyecto y utilizar los modelos y herramientas } \\
\text { disponibles. }\end{array}$ & 3 & $\mathrm{OB}$ & 1 & $\begin{array}{l}\text { Teórica- } \\
\text { Aplicada }\end{array}$ \\
\hline $\begin{array}{l}\text { Marketing y estudios de } \\
\text { usuarios }\end{array}$ & $\begin{array}{l}\text { Conocer y aplicar los principales modelos y técnicas en el campo del } \\
\text { marketing y los estudios de usuarios aplicados a la gestión de unidades } \\
\text { y servicios de información y documentación. }\end{array}$ & 3 & OB & 1 & $\begin{array}{l}\text { Teórica- } \\
\text { Aplicada }\end{array}$ \\
\hline $\begin{array}{l}\text { Evaluación de unidades y } \\
\text { servicios de información }\end{array}$ & $\begin{array}{l}\text { Comprender la importancia de la evaluación unidades y servicios de } \\
\text { información. Conocer y aplicar los diferentes modelos y métodos de } \\
\text { evaluación disponibles. }\end{array}$ & 3 & OB & 1 & $\begin{array}{l}\text { Teórica- } \\
\text { Aplicada }\end{array}$ \\
\hline $\begin{array}{l}\text { Conservación de } \\
\text { documentos }\end{array}$ & $\begin{array}{l}\text { Conocer y aplicar los principios, técnicas y herramientas de } \\
\text { conservación preventiva de documentos en diferentes tipos de } \\
\text { unidades y servicios de información y documentación. }\end{array}$ & 3 & $\mathrm{OB}$ & 1 & $\begin{array}{l}\text { Teórica- } \\
\text { Aplicada }\end{array}$ \\
\hline $\begin{array}{l}\text { Gestión del patrimonio } \\
\text { bibliográfico y } \\
\text { documental }\end{array}$ & $\begin{array}{l}\text { Ser capaz de gestionar la adquisición, enajenación, préstamo, uso, } \\
\text { reproducción de los elementos constitutivos del Patrimonio Bibliográfico } \\
\text { y Documental; y de valorar y tasar los elementos constitutivos del } \\
\text { Patrimonio Bibliográfico y Documental. }\end{array}$ & 3 & $\mathrm{OB}$ & 1 & $\begin{array}{l}\text { Teórica- } \\
\text { Aplicada }\end{array}$ \\
\hline $\begin{array}{l}\text { Representación, } \\
\text { organización y gestión } \\
\text { del conocimiento }\end{array}$ & $\begin{array}{l}\text { Conocer, seleccionar, adecuar y evaluar los modelos teóricos y } \\
\text { aplicados para la representación, organización y gestión del } \\
\text { conocimiento en la resolución de problemas habituales en las unidades } \\
\text { y servicios de información y documentación. }\end{array}$ & 3 & $\mathrm{OB}$ & 1 & $\begin{array}{l}\text { Teórica- } \\
\text { Aplicada }\end{array}$ \\
\hline $\begin{array}{l}\text { Recursos de información } \\
\text { digital }\end{array}$ & $\begin{array}{l}\text { Saber planificar, diseñar y gestionar bases de datos documentales y } \\
\text { colecciones de documentos digitales, y aplicar los métodos y técnicas } \\
\text { correspondientes }\end{array}$ & 3 & OB & 2 & $\begin{array}{l}\text { Teórica- } \\
\text { Aplicada }\end{array}$ \\
\hline Gestión de contenidos & $\begin{array}{l}\text { Conocer y aplicar los principios, técnicas y herramientas de gestión de } \\
\text { contenidos en diferentes tipos de unidades y servicios de información y } \\
\text { documentación. }\end{array}$ & 3 & OB & 2 & $\begin{array}{l}\text { Teórica- } \\
\text { Aplicada }\end{array}$ \\
\hline $\begin{array}{l}\text { Edición y publicación } \\
\text { electrónica }\end{array}$ & $\begin{array}{l}\text { Conocer y aplicar los principios, técnicas y herramientas de la edición y } \\
\text { la publicación electrónica en diferentes tipos de unidades y servicios de } \\
\text { información y documentación. }\end{array}$ & 3 & $\mathrm{OB}$ & 2 & $\begin{array}{l}\text { Teórica- } \\
\text { Aplicada }\end{array}$ \\
\hline $\begin{array}{l}\text { Formación de usuarios y } \\
\text { alfabetización } \\
\text { informacional }\end{array}$ & $\begin{array}{l}\text { Analizar los recursos de información existentes en una unidad de } \\
\text { información, y conocer los modelos, las técnicas y procedimientos para } \\
\text { transmitirlos de forma eficiente }\end{array}$ & 3 & OB & 2 & $\begin{array}{l}\text { Teórica- } \\
\text { Aplicada }\end{array}$ \\
\hline $\begin{array}{l}\text { Relaciones y recursos } \\
\text { humanos en unidades de } \\
\text { información }\end{array}$ & $\begin{array}{l}\text { Comprender, discernir y resolver problemas de relaciones y recursos } \\
\text { humanos en servicios y unidades de información y documentación }\end{array}$ & 3 & OB & 2 & $\begin{array}{l}\text { Teórica- } \\
\text { Aplicada }\end{array}$ \\
\hline $\begin{array}{l}\text { Archivos y fondos de la } \\
\text { administración estatal y } \\
\text { autonómica }\end{array}$ & $\begin{array}{l}\text { Conocer la historia y organización de la institución productora, así } \\
\text { como tipologías de fondos y documentos, y los modelos, normas y } \\
\text { técnicas para su gestión. }\end{array}$ & 3 & OP & 2 & Aplicada \\
\hline $\begin{array}{l}\text { Archivos y fondos de la } \\
\text { administración local }\end{array}$ & $\begin{array}{l}\text { Conocer la historia y organización de la institución productora, así } \\
\text { como tipologías de fondos y documentos, y los modelos, normas y } \\
\text { técnicas para su gestión. Se estudian los archivos de diputaciones } \\
\text { provinciales, municipales, comarcales y de mancomunidades. }\end{array}$ & 3 & OP & 2 & Aplicada \\
\hline $\begin{array}{l}\text { Archivos y fondos de } \\
\text { organizaciones } \\
\text { empresariales }\end{array}$ & $\begin{array}{l}\text { Conocer la historia y organización de la institución productora, así } \\
\text { como tipologías de fondos y documentos, y los modelos, normas y } \\
\text { técnicas para su gestión. }\end{array}$ & 3 & OP & 2 & Aplicada \\
\hline Archivos y fondos & $\begin{array}{l}\text { Conocer la historia y organización de la institución productora, así } \\
\text { como tipologías de fondos y documentos, y los modelos, normas y }\end{array}$ & 3 & OP & 2 & Aplicada \\
\hline
\end{tabular}




\begin{tabular}{|c|c|c|c|c|c|}
\hline privados & $\begin{array}{l}\text { técnicas para su gestión. Se estudian los archivos eclesiásticos, } \\
\text { nobiliarios, personales, patrimoniales y familiares, y de entidades y } \\
\text { asociaciones. }\end{array}$ & & & & \\
\hline Paleografía y Diplomática & $\begin{array}{l}\text { Conocer, datar e identificar los distintos sistemas escrituarios. Estudiar } \\
\text { y analizar las características de los distintos tipos documentales. }\end{array}$ & 3 & OP & 2 & Aplicada \\
\hline Bibliotecas públicas & $\begin{array}{l}\text { Comprender el concepto, contexto, objetivos, marco jurídico y } \\
\text { normativo, las funciones y procedimientos de las bibliotecas públicas, } \\
\text { con especial atención a la gestión de los principales elementos que las } \\
\text { definen: las colecciones, los servicios, los usuarios, las tecnologías y su } \\
\text { inserción en los sistemas bibliotecarios }\end{array}$ & 3 & OP & 2 & Aplicada \\
\hline $\begin{array}{l}\text { Bibliotecas universitarias } \\
\text { y de investigación }\end{array}$ & $\begin{array}{l}\text { Comprender el concepto, contexto, objetivos, marco jurídico y } \\
\text { normativo, las funciones y procedimientos de las bibliotecas } \\
\text { universitarias y de investigación, con especial atención a la gestión de } \\
\text { los principales elementos que las definen: las colecciones, los servicios, } \\
\text { los usuarios, las tecnologías y su inserción en los sistemas } \\
\text { bibliotecarios }\end{array}$ & 3 & $\mathrm{OP}$ & 2 & Aplicada \\
\hline $\begin{array}{l}\text { Bibliotecas escolares, } \\
\text { infantiles y juveniles }\end{array}$ & $\begin{array}{l}\text { Comprender el concepto, contexto, objetivos, marco jurídico y } \\
\text { normativo, las funciones y procedimientos de las bibliotecas escolares, } \\
\text { infantiles y juveniles, con especial atención a la gestión de los } \\
\text { principales elementos que las definen: las colecciones, los servicios, los } \\
\text { usuarios, las tecnologías y su inserción en los sistemas bibliotecarios }\end{array}$ & 3 & OP & 2 & Aplicada \\
\hline $\begin{array}{l}\text { Bibliotecas y colecciones } \\
\text { históricas }\end{array}$ & $\begin{array}{l}\text { Comprender el concepto, contexto, objetivos, marco jurídico y } \\
\text { normativo, las funciones y procedimientos de las bibliotecas y } \\
\text { colecciones históricas, con especial atención a la gestión de los } \\
\text { principales elementos que las definen: las colecciones, los servicios, los } \\
\text { usuarios, las tecnologías y su inserción en los sistemas bibliotecarios }\end{array}$ & 3 & $\mathrm{OP}$ & 2 & Aplicada \\
\hline $\begin{array}{l}\text { Bibliografía e historia del } \\
\text { libro }\end{array}$ & $\begin{array}{l}\text { Comprender la importancia de la materialidad del libro antiguo, } \\
\text { relacionando la historia del libro con su materialidad. Conocer y aplicar } \\
\text { la técnica de descripción del libro antiguo. }\end{array}$ & 3 & OP & 2 & Aplicada \\
\hline $\begin{array}{l}\text { Documentación médica y } \\
\text { científico-técnica }\end{array}$ & $\begin{array}{l}\text { Conocer y aplicar los modelos, normas y técnicas de gestión de centros } \\
\text { de documentación médica y científico técnica. Aprender a utilizar con } \\
\text { eficiencia las diversas herramientas de búsqueda de información } \\
\text { médica y científica en el contexto adecuado. }\end{array}$ & 3 & OP & 2 & Aplicada \\
\hline $\begin{array}{l}\text { Documentación jurídica y } \\
\text { económico-empresarial }\end{array}$ & $\begin{array}{l}\text { Conocer y aplicar los modelos, normas y técnicas de gestión de centros } \\
\text { de documentación jurídica y económico-empresarial. Aprender a utilizar } \\
\text { con eficiencia las diversas herramientas de búsqueda de información } \\
\text { jurídica y económico-empresarial en el contexto adecuado. }\end{array}$ & 3 & OP & 2 & Aplicada \\
\hline $\begin{array}{l}\text { Documentación } \\
\text { administrativa }\end{array}$ & $\begin{array}{l}\text { Conocer y aplicar los modelos, normas y técnicas de gestión de centros } \\
\text { de documentación administrativa. Aprender a utilizar con eficiencia las } \\
\text { diversas herramientas de búsqueda de información administrativa en el } \\
\text { contexto adecuado. }\end{array}$ & 3 & $\mathrm{OP}$ & 2 & Aplicada \\
\hline $\begin{array}{l}\text { Documentación } \\
\text { informativa }\end{array}$ & $\begin{array}{l}\text { Conocer y aplicar los modelos, normas y técnicas de gestión de centros } \\
\text { de documentación informativa. Aprender a utilizar con eficiencia las } \\
\text { diversas herramientas de búsqueda de información informativa en el } \\
\text { contexto adecuado. }\end{array}$ & 3 & OP & 2 & Aplicada \\
\hline $\begin{array}{l}\text { Documentación en } \\
\text { Humanidades y } \\
\text { patrimonio histórico }\end{array}$ & $\begin{array}{l}\text { Conocer y aplicar los modelos, normas y técnicas de gestión de centros } \\
\text { de documentación en Humanidades y patrimonio histórico. Aprender a } \\
\text { utilizar con eficiencia las diversas herramientas de búsqueda de } \\
\text { información en Humanidades y patrimonio histórico en el contexto } \\
\text { adecuado. }\end{array}$ & 3 & OP & 2 & Aplicada \\
\hline Proyecto de investigación & $\begin{array}{l}\text { Planificación de un proyecto integrado de prácticas, trabajos de } \\
\text { investigación y tesis de maestría. }\end{array}$ & 5 & $\mathrm{OB}$ & 3 & Proyecto \\
\hline Prácticas especializadas & $\begin{array}{l}\text { Realización de prácticas de gestión de unidades de información y } \\
\text { documentación en centros incluidos en el programa de prácticas del } \\
\text { Máster conforme a los convenios establecidos con la Universidad. }\end{array}$ & 15 & $\mathrm{OB}$ & 3 & Prácticum \\
\hline Trabajos de investigación & $\begin{array}{l}\text { Desarrollo de uno o varios trabajos de investigación científica en } \\
\text { Información y Documentación relacionadas con cinco asignaturas del } \\
\text { máster a elección del estudiante dentro de un programa coherente de } \\
\text { investigación. }\end{array}$ & 25 & OP & $\begin{array}{l}3 \\
1 \\
4\end{array}$ & $\begin{array}{l}\text { Proyecto de } \\
\text { investigación }\end{array}$ \\
\hline Tesis de maestría & $\begin{array}{l}\text { Desarrollo de un proyecto de evaluación, mejora o diseño de un } \\
\text { servicio o unidad de información que integre la actividad de } \\
\text { investigación y la experiencia profesional obtenida en el máster. }\end{array}$ & 15 & $\mathrm{OB}$ & 4 & Proyecto \\
\hline
\end{tabular}

